\title{
MIXING OF PHASES IN NON-CRYSTALLINE MATERIALS: APPLICATION TO CARBON
}

\author{
G.C. SUMMERFIELD
}

Nuclear Engineering Department, University of Michigan, Ann Arbor, Michigan 48I09, USA

\author{
D.F.R. MILDNER
}

Research Reactor Facility and Department of Physics, University of Missouri, Columbia, Missouri 65211, USA

\section{J.M. CARPENTER}

Intense Pulsed Neutron Source, Argonne National Laboratory, Argonne, Illinois 60439, USA

Received 9 September 1982

Revised manuscript received 10 February 1983

The atomic structure of non-crystalline solids is described by the correlation function. When more than one phase is present, the simple addition of the correlation functions for the various phases in proportion to their concentrations within the system is not valid, except in the case of the completely phase-separated system. The overall correlation function must reflect the boundaries between the various phases within the system. The cross correlation functions between the phases have a dependence both on the degree of mixing of the phases and also on the sizes of the domains of the phases. The correlation function for a non-crystalline solid with more than one phase has been derived in a general way. Particular application has been made to non-crystalline carbon for which the correlation function given previously did not have the correct limits because of absence of the cross correlation terms.

\section{Introduction}

The atomic structure of non-crystalline materials is of ten expressed by the correlation function $g(\boldsymbol{r})$, which is defined as the atom density at a distance $\boldsymbol{r}$ from an atom located at the origin, averaged over all origin atoms in the system. This function is equivalent to the Patterson function used for crystalline materials, and is related to the scattering cross section $S(Q)$ of the system through a Fourier transform. Since non-crystalline materials do not have long-range order as in crystals, this function $g(r)$ has peaks which become less distinct and more broad with increasing distance $r$, until $g(r)$ approaches a constant value equal to $g_{o}$ which is the average atom density in the system. For crystalline materials which have more than one phase or crystal structure, one must use a combination of several Patterson functions. For non-crystalline 
materials which have more than one phase, correlation functions corresponding to each distinct phase must be summed in that fashion which reflects the mode of mixing of the various phases. The simple addition of the various correlation functions in proportion to their concentrations within the system is only valid for systems with completely separated phases. In general, there should be cross correlation functions between phases which reflect the boundaries between phases within the system.

This paper derives the correlation function for a non-crystalline material with more than one phase. The system is divided up into cells, with each cell having a unique phase. The correlation functions relating the interatomic distances within each phase and cross correlation functions relating the interatomic distances between atoms in different phases are defined. In addition, a cell correlation function is defined which describes the average size and relative locations of the cells within the system. The mixing of the phases is described by a "randomness function", which has readily interpretable limits when the phases are completely randomly mixed and when the two phases are totally separated.

Though this paper derives in a general way the correlation function for a non-crystalline material with more than one phase, particular application is made to amorphous carbon, which may exist in two different phases. Previous workers [1] have presented a correlation function for amorphous carbon which has been shown to be incorrect [2]. Their method of accounting for the boundaries between phases is too simplistic, in that the function gives an absurd result in one concentration limit. We review the basis for the rejection of their correlation function before deriving the true correlation function starting from the definition of the structure factor. The method of the derivation can be used for other systems, and indeed is valid also for crystalline systems.

\section{Non-crystalline carbon}

Diffraction studies of non-crystalline carbon using electron [3,4], X-ray [5-11] or neutron $[2,12,13]$ radiation have shown that the atomic structure depends on the precursor material, the method of preparation and the heat treatment temperature [14]. These studies have demonstrated that carbons can be produced with short-range order exhibiting features which suggest that different proportions of materials with short-range order similar to that of the two crystalline forms of carbon, graphite and diamond, comprise the various materials. The proportion of diamond-like bonding is usually small [2]. The earlier diffraction studies [3-6] showed a relatively large amount of tetrahedral bonding, though there is now doubt about the validity of these results [2]. All the later diffraction studies [7-13] correspond to some extent to a model originally proposed by Noda and Inagaki [6], that of small regions of partially ordered layers containing trigonally (graphite-like) coordinated carbon atoms, 
linked by a random network of tetrahedrally (diamond-like) coordinated carbon atoms. However, none is consistent with a significant amount of tetrahedral bonding, so that the tetrahedral networks have no sizable domains.

Stenhouse and Grout [1] have investigated the properties of a model of non-crystalline carbon containing both trigonally and tetrahedrally coordinated atoms in which the size and proportion of each domain can be varied. They claim that the correlation function is given by

$$
g(r)=(1-x)\left\{\varepsilon(r) g^{\mathrm{G}}(r)+[1-\varepsilon(r)] g^{\mathrm{D}}(r)\right\}+x g^{\mathrm{D}}(r),
$$

where $g^{\mathrm{G}}(r)$ and $g^{\mathrm{D}}(r)$ are the pair correlation functions for an infinite layered graphite-like region and an infinite tetrahedrally coordinated random network respectively, $x$ is the fraction of tetrahedrally bonded atoms, and $\varepsilon(r)$ is the probability that two atoms separated by a distance $r$ lie in the same graphite-like domain. The model has been shown to fit the electron diffraction data of Kakinoki et al. [3] on thin amorphous carbon films and the X-ray diffraction data of Franklin [5] on amorphous carbon powder, using 75\% and $50 \%$ of tetrahedrally bonded carbon atoms respectively. The comparison with the neutron diffraction of Mildner and Carpenter [13] on a glassy carbon which showed little evidence for tetrahedral bonding demonstrated that the model was inadequate to explain the structure of glassy carbon, except as a degenerate case in which the amount of tetrahedral bonding is negligible $(x=0)$. In this case, the correlation function reduces to

$$
g(r)=\varepsilon(r) g^{\mathrm{G}}(r)+[1-\varepsilon(r)] g^{\mathrm{D}}(r) .
$$

A prominent feature at $18.5 \AA^{-1}$ in the structure factor of the Stenhouse-Grout model, which is present only for the model with zero tetrahedral bonding $(x=0)$, corresponds well to the broad peak found at that scattering vector in the neutron diffraction data [2].

We have pointed out [2] that there is an inconsistency in the correlation function (eq. (1)) of Stenhouse and Grout. The function $\varepsilon(r)$ is a correction factor which compensates for edge effects in a finite-sized model [15], and is therefore determined by the size and shape of the graphite-like domains, which also give rise to the shape and breadth of the diffraction peaks. For negligible tetrahedral bonding ( $x \simeq 0$ ), the correlation function is given by eq. (2). For a layered domain of infinite extent, $\varepsilon(r)=1$ for all $r$, so that the correlation function becomes $g^{\mathrm{G}}(r)$, that of single-crystal graphite. For a finite-sized model, however, $\varepsilon(r)=1$ for small $r$, but at large $r, \varepsilon(r)$ approaches zero. Hence, in the limit of large $r$, the correlation function given by eq. (1) approaches $g^{\mathrm{D}}(r)$, that of diamond. This result is independent of $x$, the amount of tetrahedral bonding, and in particular is given even when $x=0$, corresponding to no tetrahedrally bonded regions at all! Hence the correlation function of Stenhouse and Grout is not correct and a more general expression must be found for the correlation function for a system containing two phases.

In the limit of small $r$, it is reasonable to expect the correlation function to 
be given by

$$
g(r)=(1-x) g^{\mathrm{G}}(r)+x g^{\mathrm{D}}(r) .
$$

However there must be at least one other term in this expression which is negligible at small $r$, but which would give the correct limit to the correlation function at large $r$.

It may be possible to make an approximation which is better than that (eq. (1)) of Stenhouse and Grout, by introducing two functions, $\varepsilon^{\mathrm{G}}(r)$ and $\varepsilon^{\mathrm{D}}(r)$, which are the probabilities that two atoms that are separated by a distance $r$ lie in the same graphite-like and diamond-like domains respectively. We can define the correlation function by

$$
\begin{aligned}
g(r)= & (1-x)\left\{\varepsilon^{\mathrm{G}}(r) g^{\mathrm{G}}(r)+\left[1-\varepsilon^{\mathrm{G}}(r)\right] g_{0}(r)\right\} \\
& +x\left\{\varepsilon^{\mathrm{D}}(r) g^{\mathrm{D}}(r)+\left[1-\varepsilon^{\mathrm{D}}(r)\right] g_{0}(r)\right\},
\end{aligned}
$$

where

$$
g_{0}(r)=(1-x) g^{\mathrm{G}}(r)+x g^{\mathrm{D}}(r)
$$

is the average correlation function for the totally phase-separated sytem. This is similar in form to that of Stenhouse and Grout, except that they have defined the average correlation function $g_{0}(r)$ as equal to that for diamond $g^{\mathrm{D}}(r)$. By eq. (5), this also means $g^{\mathrm{G}}(r) \equiv g^{\mathrm{D}}(r)$ so that their correlation function corresponds only to one phase.

The correlation function defined by eq. (4) may be rearranged to give

$$
\begin{aligned}
g(r)= & (1-x) g^{\mathrm{G}}(r)+x g^{\mathrm{D}}(r) \\
& +x(1-x)\left[\varepsilon^{\mathrm{G}}(r)-\varepsilon^{\mathrm{D}}(r)\right]\left[g^{\mathrm{G}}(r)-g^{\mathrm{D}}(r)\right] .
\end{aligned}
$$

In the limit of small $r$, the correction factors for both phases, $\varepsilon^{\mathrm{G}}(r)$, and $\varepsilon^{\mathrm{D}}(r)$, tend to unity, so that the correlation function reduces to that of eq. (3), with the correct limits at $x=0$ and 1. In the limit of large $r$, both $\varepsilon^{\mathrm{G}}(r)$ and $\varepsilon^{\mathrm{D}}(r)$ tend to zero, so that the correlation function again reduces to eq. (3). However, this approximation may still not be good enough, since an adjacent domain is either graphite-like or diamond-like and not a domain with an average correlation function. This means that there are cross correlation terms which have been neglected, so that even eq. (6) may not go to the correct limit at large $r$.

In this paper we consider the general problem of a system that has many regions which contain one of two types of bonding or phase, and relate the system correlation function to individual and cross correlation functions. Though the treatment is presented for graphite-like and diamond-like carbon regions, the mathematics are sufficiently general, in fact, for any two phase problem of non-crystalline substances. 


\section{Theory}

Consider a system of cells, labelled by the subscript $M$, of atoms, labelled by the subscript $j$, of size sufficiently large that one can speak meaningfully about a correlation function for the atoms within each cell. The intensity of radiation scattered by the system as a whole as a function of the scattering vector $\boldsymbol{Q}$ is given by

$$
S(\boldsymbol{Q})=\left\langle\left|\sum_{\boldsymbol{M}} \sum_{j} \exp \left(\mathrm{i} \boldsymbol{Q} \cdot\left(\boldsymbol{R}_{M}+\boldsymbol{r}_{j}\right)\right)\right|^{2}\right\rangle,
$$

where $\boldsymbol{R}_{M}$ is the radius vector of the center of the $M$ th cell, and $\boldsymbol{r}_{j}$ is the radius vector of the $j$ th atom in that cell relative to its center of mass. The sums are extended over all atoms $j$ within each cell, and over all cells $M$ within the system. The brackets denote an ensemble average of all atoms within the system. The sum of squared terms over all cells can be separated into two sums, one over $M=M^{\prime}$ and the other over $M \neq M^{\prime}$. The sum over $M$ may be interchanged with the brackets denoting averaging to give

$$
\begin{aligned}
S(\boldsymbol{Q})= & \sum_{\boldsymbol{M}}\left\langle\left|\sum_{j} \exp \left(\mathrm{i} \boldsymbol{Q} \cdot \boldsymbol{r}_{j}\right)\right|^{2}\right\rangle \\
& +\sum_{M \neq M^{\prime}}\left\langle\exp \left[\mathrm{i} \boldsymbol{Q} \cdot\left(\boldsymbol{R}_{M}-\boldsymbol{R}_{\boldsymbol{M}^{\prime}}\right)\right] \sum_{j j^{\prime}} \exp \left[\mathrm{i} \boldsymbol{Q} \cdot\left(\boldsymbol{r}_{j}-\boldsymbol{r}_{j^{\prime}}\right)\right]\right\rangle .
\end{aligned}
$$

This is analogous to earlier expressions for the scattering from molecular fluids [16]. The first term is the "inner" scattering and the second the "outer" scattering, which refer respectively to interference effects within molecules and to those between distinct molecules. In the present case, we interpret the sums over $M$ to represent sums of average structure factors, and identify two types of cells, labelled $\mathrm{G}$ and $\mathrm{D}$, each with a number of cells, $n_{\mathrm{G}}$ and $n_{\mathrm{D}}$, respectively. The total number of cells is $n_{\mathrm{C}}=n_{\mathrm{G}}+n_{\mathrm{D}}$, and the proportions of $\mathrm{G}$ and $\mathrm{D}$ type cells are given by $(1-x)=n_{\mathrm{G}} /\left(n_{\mathrm{G}}+n_{\mathrm{D}}\right)$ and $x=n_{\mathrm{D}} /\left(n_{\mathrm{G}}+n_{\mathrm{D}}\right)$ respectively. We also identify a structure factor for each type of cell, given by

$$
F_{\mathrm{G}}=\sum_{j_{\mathrm{i}}} \exp \left(\mathrm{i} \boldsymbol{Q} \cdot \boldsymbol{r}_{j}\right)
$$

and

$$
F_{\mathrm{D}}=\sum_{j_{\mathrm{D}}} \exp \left(\mathrm{i} \boldsymbol{Q} \cdot \boldsymbol{r}_{j}\right) .
$$

Then the normalized differential cross-section for the material may be written

$$
\begin{aligned}
S(Q)= & (1-x) n_{\mathrm{C}} F_{\mathrm{G}}^{2}+x n_{\mathrm{C}} F_{\mathrm{D}}^{2}+\sum_{M \neq M^{\prime}} \exp \left[\mathrm{i} Q \cdot\left(\boldsymbol{R}_{M}-\boldsymbol{R}_{M^{\prime}}\right)\right] \\
& \times\left(P_{M \mathrm{G}^{\prime} M^{\prime} \mathrm{G}} F_{\mathrm{G}}^{2}+P_{M \mathrm{D} M^{\prime} \mathrm{D}} F_{\mathrm{D}}^{2}+P_{M \mathrm{G}^{\prime} \mathrm{D}^{\prime}} F_{\mathrm{G}}^{*} F_{\mathrm{D}}+P_{M M^{\prime} \mathrm{G}} F_{\mathrm{G}} F_{\mathrm{D}}^{*}\right),
\end{aligned}
$$

where the factors $P_{M \mathrm{G} M^{\prime} \mathrm{D}}, P_{M \mathrm{G} M^{\prime} \mathrm{G}}, P_{M \mathrm{D} M^{\prime} \mathrm{G}}$ and $P_{M \mathrm{D} M^{\prime} \mathrm{D}}$ are joint probabili- 
ties that cell $M$ is of type $G$ and cell $M^{\prime}$ is of type D and so on.

These probabilities may be written in terms of a randomness function $\alpha$ by the following equations:

$$
\begin{aligned}
& P_{M G M^{\prime} \mathrm{G}}=(1-x)^{2}+x(1-x) \alpha\left(\boldsymbol{R}_{M}-\boldsymbol{R}_{M^{\prime}}\right), \\
& P_{M \mathrm{D} M^{\prime} \mathrm{D}}=x^{2}+x(1-x) \alpha\left(\boldsymbol{R}_{M}-\boldsymbol{R}_{M^{\prime}}\right)
\end{aligned}
$$

and

$$
P_{M G M^{\prime} \mathrm{D}}=P_{M \mathrm{D} M^{\prime} \mathrm{G}}=x(1-x)\left[1-\alpha\left(\boldsymbol{R}_{M}-\boldsymbol{R}_{M^{\prime}}\right)\right],
$$

where $\alpha\left(\boldsymbol{R}_{M}-\boldsymbol{R}_{M}\right)$ is a parameter which expresses the deviation from randomness of the cells. Note that $\alpha\left(\boldsymbol{R}_{M}-\boldsymbol{R}_{M^{\prime}}\right)$ denotes a function rather than an algebraic expression. That there is only one function $\alpha$ follows from the requirement that

$$
P_{M \mathrm{G} M^{\prime} \mathrm{G}}+P_{M \mathrm{G} M^{\prime} \mathrm{D}}=1-x
$$

and

$$
P_{M \mathrm{D} M^{\prime} \mathrm{D}}+P_{M M^{\prime} \mathrm{D}}=x .
$$

The limits on the size of $\alpha$ can be obtained by noting that the probabilities $P_{M G M^{\prime} \mathrm{G}}, P_{M \mathrm{D}^{\prime} \mathrm{D}}$ and $P_{M G M^{\prime} \mathrm{D}}$ must be greater than zero and less than unity. These conditions require that $\alpha$ be less than unity and greater than the larger of $-x /(1-x)$ and $-(1-x) / x$. That is, $\alpha=0$ gives total randomness with the $G$ and $D$ type cells totally mixed, and $\alpha=1$ gives totally separated $G$ and $D$ phases. Substituting the values of the probabilities into eq. (10) gives

$$
\begin{aligned}
S(\boldsymbol{Q})= & (1-x) n_{\mathrm{C}} F_{\mathrm{G}}^{2}+x n_{\mathrm{C}} F_{\mathrm{D}}^{2}+\sum_{M \neq M^{\prime}} \exp \left[\mathrm{i} \boldsymbol{Q} \cdot\left(\boldsymbol{R}_{M}-\boldsymbol{R}_{M^{\prime}}\right)\right] \\
& \times\left[\left((1-x) F_{\mathrm{G}}+\left.x F_{\mathrm{D}}\right|^{2}+x(1-x) \alpha\left(\boldsymbol{R}_{M}-\boldsymbol{R}_{M^{\prime}}\right)\left|F_{\mathrm{G}}-F_{\mathrm{D}}\right|^{2}\right] .\right.
\end{aligned}
$$

We now develop some relationships which illuminate the meaning of the cell correlation functions. Let $X(\boldsymbol{r})$ be the probability that the cell at location $r$ is a D type cell. Then the joint probability that the cell at location $R \equiv \boldsymbol{R}_{M}-\boldsymbol{R}_{M^{\prime}}$ is also a $\mathrm{G}$ cell is given by

$$
P_{M M^{\prime} \mathrm{G}}=\overline{X(\boldsymbol{r})[1-X(\boldsymbol{r}+\boldsymbol{R})],}
$$

where the vinculum denotes an average taken over the entire system of volume $V$. Then the proportion of $\mathrm{D}$ type cells is given by

$$
x=\overline{X(\boldsymbol{r})}=\frac{1}{V} \int \mathrm{d}^{3} \boldsymbol{r} X(\boldsymbol{r}) .
$$

In terms of the joint probabilities, the concentration fraction $x$ is also given by

$$
\begin{aligned}
x & =P_{M M^{\prime} \mathrm{G}}+P_{M M^{\prime} \mathrm{D}} \\
& =x(1-x)-x(1-x) \alpha(\boldsymbol{R})+\overline{X(\boldsymbol{r}) X(\boldsymbol{r}+\boldsymbol{R}),}
\end{aligned}
$$


so that

$$
\alpha(\boldsymbol{R})=\frac{\overline{X(\boldsymbol{r}) X(\boldsymbol{r}+\boldsymbol{R})}}{x(1-x)}-\frac{x}{(1-x)} .
$$

This can be used as the definition of $\alpha(\boldsymbol{R})$, the randomness parameter, introduced for the definitions of the joint probabilities in eq. (11). For $\boldsymbol{R}=0$, we obtain

$$
\alpha(0)=\frac{\overline{X(r)^{2}}-\overline{X(r)}^{2}}{x(1-x)} .
$$

For $\alpha=0, \overline{X^{2}} \equiv \bar{X}^{2}$, and the phases are totally randomly mixed. For $\overline{X(\boldsymbol{r}) X(\boldsymbol{r}+\boldsymbol{R})}=x, \alpha=1$, and the two phases are totally separated, so that $P_{M M^{\prime} \mathrm{D}}=P_{M \mathrm{DM}^{\prime} \mathrm{G}}=0$ !

Now if we note that

$$
\left(F_{\mathrm{G}}^{*} F_{\mathrm{D}}+F_{\mathrm{G}} R_{\mathrm{D}}^{*}\right)=\left(F_{\mathrm{G}}^{2}+F_{\mathrm{D}}^{2}\right)-\left|F_{\mathrm{G}}-F_{\mathrm{D}}\right|^{2},
$$

then eq. (13) may be rearranged to give

$$
\begin{aligned}
& S(Q)=(1-x) n_{C} F_{\mathrm{G}}^{2}+x n_{\mathrm{C}} F_{\mathrm{D}}^{2} \\
&+\sum_{M \neq M^{\prime}}(1-x) F_{\mathrm{G}}^{2} \exp \left[\mathrm{i} \boldsymbol{Q} \cdot\left(\boldsymbol{R}_{M}-\boldsymbol{R}_{M^{\prime}}\right)\right] \\
&+\sum_{M \neq M^{\prime}} x F_{\mathrm{D}}^{2} \exp \left[\mathrm{i} \boldsymbol{Q} \cdot\left(\boldsymbol{R}_{M}-\boldsymbol{R}_{M^{\prime}}\right)\right] \\
&-\sum_{M \neq M^{\prime}} x(1-x)\left[1-\alpha\left(\boldsymbol{R}_{M}-\boldsymbol{R}_{M^{\prime}}\right)\right]\left|F_{\mathrm{G}}-F_{\mathrm{D}}\right|^{2} \exp \left[\mathrm{i} \boldsymbol{Q} \cdot\left(\boldsymbol{R}_{M}-\boldsymbol{R}_{M^{\prime}}\right)\right] .
\end{aligned}
$$

We note that the cross-sections for systems of completely G or D type cells are given by

$$
S_{\mathrm{G}}(\boldsymbol{Q})=n_{\mathrm{C}} F_{\mathrm{G}}^{2}+\sum_{M \equiv M^{\prime}} F_{\mathrm{G}}^{2} \exp \left[\mathrm{i} Q \cdot\left(\boldsymbol{R}_{M}-\boldsymbol{R}_{M^{\prime}}\right)\right]
$$

and

$$
S_{\mathrm{D}}(\boldsymbol{Q})=n_{\mathrm{C}} F_{\mathrm{D}}^{2}+\sum_{M \neq M^{\prime}} F_{\mathrm{D}}^{2} \exp \left[\mathrm{i} \boldsymbol{Q} \cdot\left(\boldsymbol{R}_{M}-\boldsymbol{R}_{M^{\prime}}\right)\right]
$$

respectively. Hence eq. (20) may be rewritten

$$
\begin{aligned}
& S(\boldsymbol{Q})=(1-x) S_{\mathrm{G}}(\boldsymbol{Q})+x S_{\mathrm{D}}(\boldsymbol{Q}) \\
& -\sum_{M \neq M^{\prime}} x(1-x)\left[1-\alpha\left(\boldsymbol{R}_{M}-\boldsymbol{R}_{M^{\prime}}\right)\right]\left|F_{\mathrm{G}}-F_{\mathrm{D}}\right|^{2} \exp \left[\mathrm{i} \boldsymbol{Q} \cdot\left(\boldsymbol{R}_{M}-\boldsymbol{R}_{M^{\prime}}\right)\right] .
\end{aligned}
$$

We now introduce the atomic density functions fo provide further insight into the formulation. The structure factor $F_{\mathrm{G}}$, for example, is defined in eq. (9), 
so that

$$
\left|F_{\mathrm{G}}\right|^{2}=\sum_{j_{G} j_{\mathrm{G}}^{\prime}} \exp \left[\mathrm{i} \boldsymbol{Q} \cdot\left(\boldsymbol{r}_{j}-\boldsymbol{r}_{j^{\prime}}\right)\right] .
$$

Consider also an atom density function $\gamma_{G}(r)$ defined by

$$
\gamma_{\mathrm{G}}(\boldsymbol{r})=\sum_{j_{\mathrm{G}}} \delta\left(\boldsymbol{r}-\boldsymbol{r}_{j}\right)
$$

which is the atom density for a $G$ type cell. Then the square of the structure factor may be expressed by

$$
\left|F_{\mathrm{G}}\right|^{2}=\int \mathrm{d}^{3} \boldsymbol{r} \int \mathrm{d}^{3} \boldsymbol{r}^{\prime} \exp \left[\mathrm{i} \boldsymbol{Q} \cdot\left(\boldsymbol{r}-\boldsymbol{r}^{\prime}\right)\right] \gamma_{\mathrm{G}}(\boldsymbol{r}) \gamma_{\mathrm{G}}\left(\boldsymbol{r}^{\prime}\right)
$$

A pair correlation function $g_{G G}(R)$ is defined for a single cell by

$$
g_{\mathrm{GG}}(\boldsymbol{R})=\int \mathrm{d}^{3} \boldsymbol{R}^{\prime} \gamma_{\mathrm{G}}\left(\boldsymbol{R}^{\prime}\right) \gamma_{\mathrm{G}}\left(\boldsymbol{R}^{\prime}+\boldsymbol{R}\right),
$$

so that eq. (25) becomes

$$
\begin{aligned}
\left|F_{\mathrm{G}}\right|^{2} & =\int \mathrm{d}^{3} \boldsymbol{R} \int \mathrm{d}^{3} \boldsymbol{r}^{\prime} \exp (\mathrm{i} \boldsymbol{Q} \cdot \boldsymbol{R}) \gamma_{\mathrm{G}}\left(\boldsymbol{r}^{\prime}+\boldsymbol{R}\right) \gamma_{\mathrm{G}}\left(\boldsymbol{r}^{\prime}\right) \\
& =\int \mathrm{d}^{3} \boldsymbol{r} \exp (\mathrm{i} \boldsymbol{Q} \cdot \boldsymbol{r}) g_{\mathrm{GG}}(\boldsymbol{r}) .
\end{aligned}
$$

Similarly

$$
\left|F_{\mathrm{D}}\right|^{2}=\int \mathrm{d}^{3} \boldsymbol{r} \exp (\mathrm{i} \boldsymbol{Q} \cdot \boldsymbol{r}) g_{\mathrm{GG}}(\boldsymbol{r}) .
$$

Also the expression $F_{\mathrm{G}} F_{\mathrm{D}}^{*}$ is given by

$$
F_{\mathrm{G}} F_{\mathrm{D}}^{*}=\sum_{j_{\mathrm{G}} j_{\mathrm{D}}^{\prime}} \exp \left[\mathrm{i} \boldsymbol{Q} \cdot\left(\boldsymbol{r}_{j}-\boldsymbol{r}_{j^{\prime}}\right)\right]
$$

Using the atom density functions $\gamma_{G}(r)$ and $\gamma_{D}(r)$ to define a pair correlation function $g_{G D}(r)$ between cells,

$$
g_{\mathrm{GD}}(\boldsymbol{r})=\int \mathrm{d}^{3} \boldsymbol{r}^{\prime} \gamma_{\mathrm{G}}\left(\boldsymbol{r}^{\prime}\right) \gamma_{\mathrm{D}}\left(\boldsymbol{r}^{\prime}+\boldsymbol{r}\right)
$$

the expression for $F_{\mathrm{G}} F_{\mathrm{D}}^{*}$ is given by

$$
\begin{aligned}
F_{\mathrm{G}} F_{\mathrm{D}}^{*} & =\int \mathrm{d}^{3} \boldsymbol{r} \int \mathrm{d}^{3} \boldsymbol{r}^{\prime} \exp \left[\mathrm{i} \boldsymbol{Q} \cdot\left(\boldsymbol{r}-\boldsymbol{r}^{\prime}\right)\right] \gamma_{\mathrm{G}}(\boldsymbol{r}) \gamma_{\mathrm{D}}\left(\boldsymbol{r}^{\prime}\right) \\
& =\int \mathrm{d}^{3} \boldsymbol{R} \int \mathrm{d}^{3} \boldsymbol{r}^{\prime} \exp (\mathrm{i} \boldsymbol{Q} \cdot \boldsymbol{R}) \gamma_{\mathrm{G}}\left(\boldsymbol{r}^{\prime}+\boldsymbol{R}\right) \gamma_{\mathrm{D}}\left(\boldsymbol{r}^{\prime}\right) \\
& =\int \mathrm{d}^{3} \boldsymbol{r} \exp (\mathrm{i} \boldsymbol{Q} \cdot \boldsymbol{r}) g_{\mathrm{DG}}(\boldsymbol{r}) .
\end{aligned}
$$


We also define the cell correlation function $G(\boldsymbol{R})$ by

$$
G(\boldsymbol{R})=\sum_{M \neq M^{\prime}} \delta\left(\boldsymbol{R}-\boldsymbol{R}_{M}+\boldsymbol{R}_{M^{\prime}}\right)
$$

so that

$$
\int \mathrm{d}^{3} \boldsymbol{R} G(\boldsymbol{R}) \exp (\mathrm{i} \boldsymbol{Q} \cdot \boldsymbol{R})=\sum_{M \neq M^{\prime}} \exp \left[\mathrm{i} \boldsymbol{Q} \cdot\left(\boldsymbol{R}_{M}-\boldsymbol{R}_{M^{\prime}}\right)\right]
$$

Substitution of all these correlation functions, $g_{\mathrm{GG}}(\boldsymbol{r}), g_{\mathrm{DD}}(\boldsymbol{r}), g_{\mathrm{GD}}(\boldsymbol{r})$, $g_{\mathrm{DG}}(\boldsymbol{r})$ and $G(\boldsymbol{R})$ into the expression for $S(Q)$ in eq. (20) gives the following result for the cross-section of the entire system:

$$
\begin{aligned}
S(\boldsymbol{Q})= & (1-x) n_{\mathrm{C}} \int \mathrm{d}^{3} \boldsymbol{r} \exp (\mathrm{i} \boldsymbol{Q} \cdot \boldsymbol{r}) g_{\mathrm{GG}}(\boldsymbol{r}) \\
& +x n_{\mathrm{C}} \int \mathrm{d}^{3} \boldsymbol{r} \exp (\mathrm{i} \boldsymbol{Q} \cdot \boldsymbol{r}) g_{\mathrm{DD}}(\boldsymbol{r}) \\
& +(1-x) \int \mathrm{d}^{3} \boldsymbol{r} \exp (\mathrm{i} \boldsymbol{Q} \cdot \boldsymbol{r}) g_{\mathrm{GG}}(\boldsymbol{r}) \int \mathrm{d}^{3} \boldsymbol{R} G(\boldsymbol{R}) \exp (\mathrm{i} \boldsymbol{Q} \cdot \boldsymbol{R}) \\
& +x \int \mathrm{d}^{3} \boldsymbol{r} \exp (\mathrm{i} \boldsymbol{Q} \cdot \boldsymbol{r}) g_{\mathrm{DD}}(\boldsymbol{r}) \int \mathrm{d}^{3} \boldsymbol{R} G(\boldsymbol{R}) \exp (\mathrm{i} \boldsymbol{Q} \cdot \boldsymbol{R}) \\
& -x(1-x) \int \mathrm{d}^{3} \boldsymbol{r} \exp (\mathrm{i} \boldsymbol{Q} \cdot \boldsymbol{r})\left[g_{\mathrm{GG}}(\boldsymbol{r})+g_{\mathrm{DD}}(\boldsymbol{r})-g_{\mathrm{GD}}(\boldsymbol{r})-g_{\mathrm{DG}}(\boldsymbol{r})\right] \\
& \times \int \mathrm{d}^{3} \boldsymbol{R} G(\boldsymbol{R}) \exp (\mathrm{i} \boldsymbol{Q} \cdot \boldsymbol{R})[1-\alpha(\boldsymbol{R})] .
\end{aligned}
$$

Performing the inverse Fourier transform gives the following result for the system correlation function:

$$
\begin{aligned}
g(\boldsymbol{r})= & (1-x) n_{\mathrm{C}} g_{\mathrm{GG}}(\boldsymbol{r})+x n_{\mathrm{C}} g_{\mathrm{DD}}(\boldsymbol{r}) \\
& +\int \mathrm{d}^{3} \boldsymbol{R} G(\boldsymbol{R})\left\{(1-x) g_{\mathrm{GG}}(\boldsymbol{r}-\boldsymbol{R})+x g_{\mathrm{DD}}(\boldsymbol{r}-\boldsymbol{R})\right\} \\
& -x(1-x) \int \mathrm{d}^{3} \boldsymbol{R} G(\boldsymbol{R})[1-\alpha(\boldsymbol{R})]\left[g_{\mathrm{GG}}(\boldsymbol{r}-\boldsymbol{R})\right. \\
& \left.+g_{\mathrm{DD}}(\boldsymbol{r}-\boldsymbol{R})-g_{\mathrm{GD}}(\boldsymbol{r}-\boldsymbol{R})-g_{\mathrm{DG}}(\boldsymbol{r}-\boldsymbol{R})\right] .
\end{aligned}
$$

In terms of the correlation functions for networks of infinite extent,

$$
g^{\mathrm{G}}(\boldsymbol{r})=n_{\mathrm{C}} g_{\mathrm{GG}}(\boldsymbol{r})+\int \mathrm{d}^{3} \boldsymbol{R} g_{G G}(\boldsymbol{r}-\boldsymbol{R}) G(\boldsymbol{R})
$$

and

$$
g^{\mathrm{D}}(\boldsymbol{r})=n_{\mathrm{C}} g_{\mathrm{DD}}(\boldsymbol{r})+\int \mathrm{d}^{3} \boldsymbol{R} g_{\mathrm{DD}}(\boldsymbol{r}-\boldsymbol{R}) G(\boldsymbol{R}),
$$


this last result can be expressed as

$$
\begin{aligned}
g(\boldsymbol{r})= & (1-x) g^{\mathrm{G}}(\boldsymbol{r})+x g^{\mathrm{D}}(\boldsymbol{r}) \\
& -x(1-x) \int \mathrm{d}^{3} \boldsymbol{R} G(\boldsymbol{R})[1-\alpha(\boldsymbol{R})]\left[g_{\mathrm{GG}}(\boldsymbol{r}-\boldsymbol{R})\right. \\
& \left.+g_{\mathrm{DD}}(\boldsymbol{r}-\boldsymbol{R})-g_{\mathrm{GD}}(\boldsymbol{r}-\boldsymbol{R})-g_{\mathrm{DG}}(\boldsymbol{r}-\boldsymbol{R})\right] .
\end{aligned}
$$

Notice that the system correlation function $g(\boldsymbol{r})$ is expressed in terms of $g^{\mathrm{G}}(\boldsymbol{r})$ and $g^{\mathrm{D}}(\boldsymbol{r})$, the correlation functions for graphite-like and diamond-like regions of infinite extent, in proportion to their abundance, plus a term that expresses cross correlations, which will be shown to give rise to small-angle scattering.

\section{Discussion}

We now consider various limiting cases as examples. For $x=0, g(\boldsymbol{r})=g^{\mathrm{G}}(\boldsymbol{r})$, the correlation function for an infinite cell having graphite-like order. Similarly for $x=1, g(r)=g^{\mathrm{D}}(\boldsymbol{r})$, the correlation function for an infinite cell with diamond-like order. For $\alpha=1, g(\boldsymbol{r})=(1-x) g^{\mathrm{G}}(\boldsymbol{r})+x g^{\mathrm{D}}(\boldsymbol{r})$, the correlation function for a totally phase-separated system, and is equivalent to eq. (3). For the case of $\alpha=0$, the system comprising totally randomly mixed cells of types $\mathrm{G}$ and $\mathrm{D}$, the correlation function is given by

$$
\begin{aligned}
g(\boldsymbol{r})= & (1-x) n_{\mathrm{C}} g_{\mathrm{GG}}(\boldsymbol{r})+x n_{\mathrm{C}} g_{\mathrm{DD}}(\boldsymbol{r}) \\
& +\int \mathrm{d}^{3} \boldsymbol{R} G(\boldsymbol{R})\left\{(1-x)^{2} g_{\mathrm{GG}}(\boldsymbol{r}-\boldsymbol{R})+x^{2} g_{\mathrm{DD}}(\boldsymbol{r}-\boldsymbol{R})\right. \\
& \left.+x(1-x)\left[g_{\mathrm{GD}}(\boldsymbol{r}-\boldsymbol{R})+g_{\mathrm{DG}}(\boldsymbol{r}-\boldsymbol{R})\right]\right\} .
\end{aligned}
$$

The difference between the approximation given by eq. (3) and the correct correlation function for the two mixed phases (eq. 37)) is $F(r)$, where

$$
\begin{aligned}
F(\boldsymbol{r})= & x(1-x) \int \mathrm{d}^{3} \boldsymbol{R} G(\boldsymbol{R})[1-\alpha(\boldsymbol{R})]\left[g_{\mathrm{GG}}(\boldsymbol{r}-\boldsymbol{R})+g_{\mathrm{DD}}(\boldsymbol{r}-\boldsymbol{R})\right. \\
& \left.-g_{\mathrm{GD}}(\boldsymbol{r}-\boldsymbol{R})-g_{\mathrm{DG}}(\boldsymbol{r}-\boldsymbol{R})\right] .
\end{aligned}
$$

This difference is largest for the totally randomly mixed case $(\alpha=0)$, and zero for the totally phase-separated system $(\alpha=1)$. Its evaluation requires knowledge of the cell correlation function $G(R)$, i.e. the average size and relative locations of the cells of the system.

We need to know how all the functions introduced in section 3 depend on the distance $r$. The composite correlation function $g(r)$ is defined in eq. (37) by the individual infinite correlation functions of each phase in proportion to their relative amounts found in the composite, plus a cross correlation term which is written in terms of a cell size $R$ and which depends on the cell correlation function $G(\boldsymbol{R})$, on the pair correlation functions between cells and on a randomness function $\alpha(\boldsymbol{R})$. The pair correlation function between cells is 


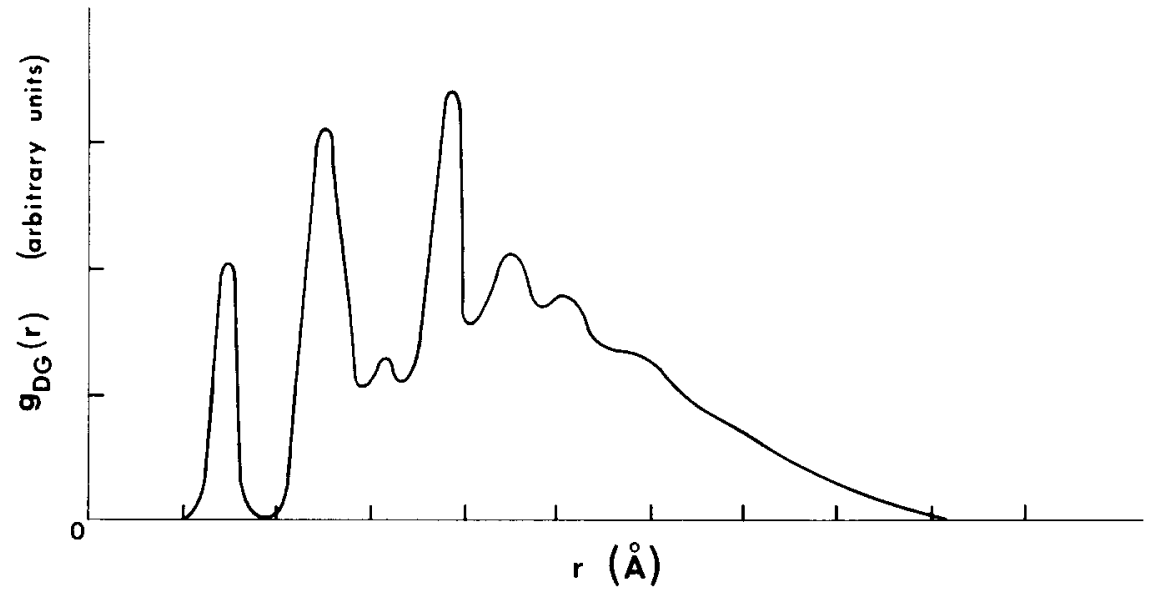

Fig. 1. A schematic diagram of an atom correlation function $g_{\mathrm{DG}}(r)$ for a cell as a function of $r$. The distances between peaks are, of course, typical atomic spacings. $g_{\mathrm{DG}}$ must go to zero as $r$ becomes large compared with a cell size. The functions $g_{\mathrm{GG}}$ and $g_{\mathrm{DD}}$ will be qualitatively similar.

defined by eq. (30), and may be described by a typical schematic diagram shown in fig. 1. Note that the functions $g_{\mathrm{DD}}(r), g_{\mathrm{GG}}(r)$ and $g_{\mathrm{DG}}(r)$ are correlation functions for atoms within cells, and therefore tend to zero for $r$ large compared with the cell dimensions.

The cell correlation function $G(R)$ depends on the size $R_{0}$ of the typical cell, and a schematic diagram is shown in fig. 2. This function is zero for small values of $R$, rises to a peak at $R=R_{0}$, and for $R \gg R_{0}$ goes to a value of $n_{C} / V$, where $V$ is the volume of a typical cell.

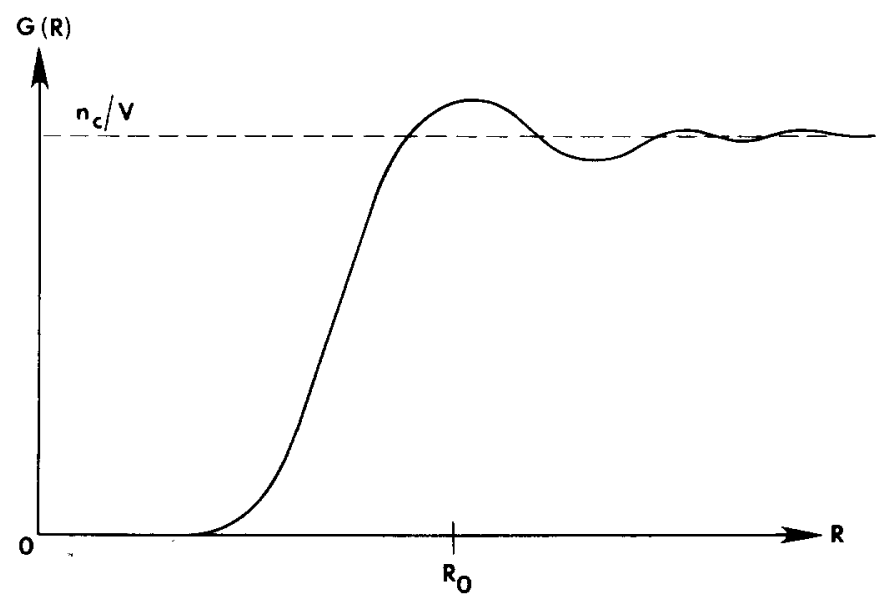

Fig. 2. A schematic diagram of the cell-cell correlation function $G(\boldsymbol{R})$. At large $R$ this function goes to the constant $n_{\mathrm{c}} / V$. 
The various cross correlation functions in eq. (37) may be viewed schematically by considering $F(\boldsymbol{r})$. The $g_{\mathrm{GG}}, g_{\mathrm{DD}}$ and $g_{\mathrm{GD}}$ functions are defined as non zero only within individual cells, and therefore at small $r$, whereas $G(\boldsymbol{R})$ is zero at small values of $\boldsymbol{R}$. Hence, $F(0)=0$. At large $\boldsymbol{r}, F(\boldsymbol{r})$ becomes

$$
\begin{aligned}
F(\boldsymbol{r})= & x(1-x) G(\boldsymbol{r})[1-\alpha(\boldsymbol{r})] \int \mathrm{d}^{3} \boldsymbol{R}\left[g_{\mathrm{GG}}(\boldsymbol{R})+g_{\mathrm{DD}}(\boldsymbol{R})\right. \\
& \left.-g_{\mathrm{GD}}(\boldsymbol{R})-g_{\mathrm{DG}}(\boldsymbol{R})\right] .
\end{aligned}
$$

The volume integrals of $g_{\mathrm{GG}}, g_{\mathrm{DD}}$ and $g_{\mathrm{GD}}$ are $N_{\mathrm{G}}^{2}, N_{\mathrm{D}}^{2}$ and $N_{\mathrm{G}} N_{\mathrm{D}}$ respectively, where $N_{\mathrm{G}}$ and $N_{\mathrm{D}}$ are the number of atoms within a $\mathrm{G}$ and D cell. Hence $F(\boldsymbol{r})$ is a functional only of $G(\boldsymbol{r})[1-\alpha(\boldsymbol{r})]$. At large $\boldsymbol{r}, \alpha(\boldsymbol{r})$ tends to zero, and $G(\boldsymbol{r})$ goes to $n_{\mathrm{c}} / V$; hence $F(\infty)=x(1-x)\left(N_{\mathrm{G}}-N_{\mathrm{D}}\right)^{2} n_{\mathrm{c}} / V$. This function will be zero at small $r$, have oscillations characteristic of $g_{\mathrm{GG}}, g_{\mathrm{DD}}$ and $g_{\mathrm{GD}}$, though modified by $G(\boldsymbol{r})$, and will rise to a value of $x(1-x)\left(N_{\mathrm{G}}-N_{\mathrm{D}}\right)^{2} n_{\mathrm{c}} / V$. This is shown schematically in fig. 3 .

The values of the correlations function for infinite regions, $g^{\mathrm{G}}(\boldsymbol{r})$ and $g^{\mathrm{D}}(\boldsymbol{r})$, at large values of $\boldsymbol{r}$ are given by $N_{\mathrm{G}}^{2} n_{\mathrm{c}} / V$ and $N_{\mathrm{D}}^{2} n_{\mathrm{c}} / V$ respectively. Hence the approximation given by eq. (3) at large $r$ is $\left[(1-x) N_{\mathrm{G}}^{2}+x N_{\mathrm{D}}^{2}\right] n_{\mathrm{c}} / V$. On the other hand, the inclusion of the cross correlation term $F(\boldsymbol{r})$ gives $\left[(1-x) N_{\mathrm{G}}+x N_{\mathrm{D}}\right]^{2} n_{\mathrm{c}} / V$ for $g(r)$ (eq. (37)) at large $\boldsymbol{r}$. We conclude that the correlation function given by eq. (6) is not strictly correct because the cross correlation term has been neglected.

Since the function $F(\boldsymbol{r})$ is nonzero only at large $\boldsymbol{r}$, the Fourier transform of

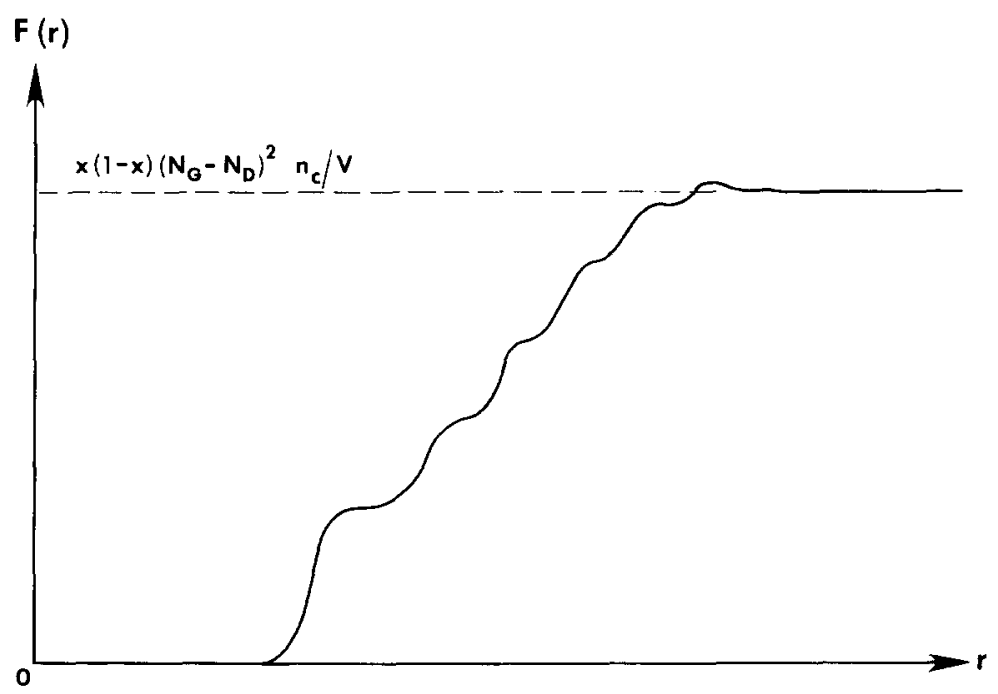

Fig. 3. A schematic diagram of the cross correlation function $F(r)$ as a function of $r$. This function is zero at small $r$, has oscillations characteristic of the atom correlation functions at intermediate values of $r$, and will go to $x(1-x)\left(N_{\mathrm{G}}-N_{\mathrm{D}}\right)^{2} n_{\mathrm{c}} / V$ at large $r$. 
$F(\boldsymbol{r})$ will only produce scattering at small angles. Then if the small-angle scattering can be removed correctly from the experimental data, the effective $g(\boldsymbol{r})$ that is determined will be given by eq. (3) to a good approximation. We have not included explicitly the effect of pores or voids in these calculations, though we know that there will be pores in any real carbon system. We should point out that pores will also contribute only to small-angle scattering and, to the extent that the small-angle scattering can be removed, will not change the validity of eq. (3).

\section{Conclusions}

The correlation function for a non-crystalline material with more than one phase has been derived from first principles. It has been shown that, except for the completely phase-separated system, the simple addition of the correlation functions of the various phases in proportion to their concentrations within the solid is incorrect. There is also a term which expresses the cross correlation between the two phases, and this term depends both on the abundances of the two phases and on their degree of mixing. This cross-correlation term gives rise to small-angle scattering in the experimental diffraction pattern.

Some immediate observations can be made regarding the application of the theory developed in this paper. The diffraction data of an amorphous system which is known to have two phases should be normalized to the correct density. An improper normalization of the experimental data can produce a large effect in $g(r)$ and errors in the measured coordination numbers. The usual procedure [17] is based on the effective exclusion of an atom from the immediate neighborhood of another atom, so that for distances $r$ less than the closest interatomic distance, the correlation function $g(r)$ is zero. This has the same effect as normalizing the structure-independent differential cross-section $(S(Q)$ at large $Q)$ with respect to some average value $g_{0}$ of the atom number density. The question is what is this average value. It is related to the value of $g(r)$ at large $r$. The Stenhouse-Grout model gives $g^{\mathrm{D}}$, which is obviously incorrect. The value given by eq. (5), which is the average of the densities of the two phases in proportion to their abundances, is valid provided that the small-angle scattering has been removed from the experimental data. In the general case, where there are correlations between the two phases, the correct value of $g_{0}$ is given by

$$
g_{0}=\left[(1-x) \sqrt{g^{\mathrm{G}}}+x \sqrt{g^{\mathrm{D}}}\right]^{2} .
$$

This result obviously has the right values in the limits for $x=0$ and $x=1$, and includes an additional cross term for arbitrary $x$.

It is also possible to examine the effect of the pores within the scope of this paper by treating the carbon material as one phase and voids as a second phase of this two-phase problem. Assume that all cells with atoms can be treated as 
$G$ cells (now labelled $C$ ), and that all $D$ cells have no atoms within them, so that there is no differentiation of bonding between domains. This means that the typical pore size is greater than that of the domains. Hence, the pair correlation function $g_{G G}(r) \equiv g_{C C}(r)$ exists, whereas the functions $g_{D D}(r)=$ $g_{\mathrm{DG}}(\boldsymbol{r})=g_{\mathrm{GD}}(\boldsymbol{r}) \equiv 0$. Then the system correlation function (eq. (37)) reduces to

$$
g(\boldsymbol{r})=(1-\phi) g^{\mathrm{C}}(\boldsymbol{r})-\phi(1-\phi) \int \mathrm{d}^{3} \boldsymbol{R} G(\boldsymbol{R})[1-\boldsymbol{\alpha}(\boldsymbol{R})] g_{\mathrm{CC}}(\boldsymbol{r}-\boldsymbol{R}),
$$

where $\phi$ is the porosity of the system, so that $(1-\phi)$ is the volume fraction of the material, and $g^{C}(r)$ is the correlation function of this composite carbon material of infinite extent. The parameter $\alpha$ is interpreted as a randomness function of the pores. The second term in eq. (42) corresponds to the small-angle scattering caused by the pore system. If this is subtracted from the experimental data, the correlation function is that of the composite material, modified by the factor $(1-\phi)$ which can be neglected by suitable normalization of the scattered intensity at large scattering vectors [13].

For the case where there are pores within the system, the normalization procedure requires the value of $g_{0}$ to be $g^{C}$ (the microscopic density) if the small-angle scattering is subtracted or not measured, or $(1-\phi) g^{\mathrm{C}}$ (the macroscopic density) if it is included. The value of $g_{0}$ which is strictly compatible with the experimental neutron diffraction data on glassy carbon [13] is not well-known. The macroscopic density is influenced by a large-scale ( $>250 \AA$ ) pore system, which gives rise to small-angle scattering in the range $Q<0.025$ $\AA^{-1}$ which was not observed. Various density measurements have been reported [18] for this particular carbon, $0.923 \mathrm{gcm}^{-3}$ bulk density, $1.41 \mathrm{gcm}^{-3}$ by helium pycnometry and $1.49 \mathrm{gcm}^{-3}$ by mercury intrusion porosimetry. These are all smaller than the microscopic crystallographic density $(2.25$ $\mathrm{gcm}^{-3}$ ) of graphite. Since the experimental data include measurement of the scattering for $Q>0.065 \AA^{-1}$ due to small-scale voids, the value of $g_{0}$ chosen for normalization is that obtained by mercury intrusion. That this is a reasonable choice may be seen from the transform of the small-angle scattering data [13]. This function gives a local density $\left(2.16 \mathrm{gcm}^{-3}\right)$ very close to that expected for crystalline graphite, whereas it far from the value for diamond $\left(3.5 \mathrm{gcm}^{-3}\right)$. This is further evidence that glassy carbon contains at most only a small fraction of tetrahedrally coordinated carbon atoms.

\section{References}

[1] B.J. Stenhouse and P.J. Grout, J. Non-Crystalline Solids 27 (1978) 247.

[2] D.F.R. Mildner and J.M. Carpenter, J. Non-Crystalline Solids 47 (1982) 391.

[3] J. Kakinoki, K. Katada, T. Hanawa and T. Ino, Acta Crystallogr. 13 (1959) 171.

[4] J. Kakinoki, K. Katada and T. Hanawa, Acta Crystallogr. 13 (1960) 448. 
[5] R.E. Franklin, Acta Crystallogr. 3 (1950) 107.

[6] T. Noda and M. Inagaki, Bull. Chem. Soc. Japan, 37 (1964) 1534.

[7] R.W. Lindberg, M.Sc. thesis, Stanford University (1969).

[8] A.C. Wright, D. Phil. thesis, University of Bristol (1969).

[9] P.E. Duwez, private communication (1971).

[10] S. Ergun and R.R. Schehl, Carbon 11 (1973) 127.

[11] G.D. Wignall and C.J. Pings, Carbon 12 (1974) 51.

[12] E.A. Lorch, unpublished (1968), via private communication, A.J. Leadbetter and A.C. Wright (1974)

[13] D.F.R. Mildner and J.M. Carpenter, in:Proc. Int. Conf. on Amorphous and Liquid Semiconductors, 1973 (North-Holland, Amsterdam, 1974) p. 463.

[14] T. Noda, M. Inagaki and S. Yamada, J. Non-Crystalline Solids 1 (1969) 285.

[15] R.J. Bell, Nature 218 (1968) 985.

[16] See, for example, W. Marshall and S.W. Lovesey, Theory of Thermal Neutron Scattering (Oxford University Press, Oxford, 1971).

[17] J. Krogh-Moe, Acta Crystallogr. 9 (1956) 951.

[18] E.E. Hucke, private communications $(1972,1973)$. 